\title{
DIAGNÓSTICO FRACTAL Y EUCLIDIANO DE CÉLULAS DE CUELLO UTERINO
}

Javier Rodríguez Velásquez MD*, Signed Prieto**, Fernando Polo MD***, Catalina Correa**, Yolanda Soracipa**, Vanessa Blanco****, Andrés Camilo Rodríguez****

\section{Resumen}

Basados en geometría fractal se hizo un diagnóstico objetivo y reproducible de células de cuello uterino, que diferencia las normales de aquellas con lesión de bajo grado (LEIBG) o cancerígenas, identificando en forma cuantitativa las células ASCUS. Objetivo: establecer una metodología diagnóstica de las células cervicales normales y preneoplásicas con aplicación simultánea de geometría fractal y euclidiana para definir parámetros matemáticos distintivos de cada uno de dichos estados. Métodos: fotografías digitales de doce células de citologías de mujeres entre 20 y 55 años (tres normales superficiales, tres normales intermedias, tres LEIBG y tres ASCUS). Mediante un programa se calculó la dimensión fractal de tres objetos matemáticos: núcleo, citoplasma y totalidad, a partir del método box-counting; de manera simultánea se determinó el número de pixeles ocupados por la superficie de cada uno y los espacios ocupados por el borde de estos objetos en cada una de las cinco rejillas, para comparar los valores obtenidos. Resultados: al superponer las rejillas de dos y cuatro pixeles los valores de los espacios de ocupación del núcleo permiten establecer diferencias matemáticas entre los grupos de células, presentando como valores en la rejilla dos: normales superficiales (53-56), normales intermedias (75), LEIBG (120-159) y ASCUS (104-121). Conclusiones: se estableció una metodología matemática diagnóstica que diferencia estados preneoplásicos con base en medidas fractales y euclidianas simultáneas del borde del núcleo celular.

Palabras clave: diagnóstico, fractal, euclidiano, citología, cérvix.

Abreviaturas: LEIBG, lesión escamosa intraepitelial de bajo grado; ASCUS atypical squamous cells of undetermined significance (células escamosas atípicas de significado indeterminado); GF, geometría fractal.

\section{FRACTAL AND EUCLIDIAN DIAGNOSIS OF CERVIX CYTOLOGY}

\section{Abstract}

An objective and reproducible diagnosis of cervix cytology was made based on fractal geometry, to discriminate between normal cells and low-grade lesion (LSIL) or cancer cells, quantitatively identifying ASCUS cells. Objective: to establish a diagnostic methodology of cervix normal cells and pre-neoplastic cells applying fractal and Euclidian geometry simultaneously to define distinctive mathematical parameters of each of these states. Methods: digital photographs of twelve cytology smears of women between 20 and 55 years of age (three normal-appearing superficial cells, three

Fecha recibido: julio 16 de 2013 - Fecha aceptado: agosto 2 de 2013

* Director Grupo Insight. Fundación Universitaria de Ciencias de la Salud. Centro de Investigaciones de la Clínica del Country. Bogotá DC, Colombia.

** Investigadora Grupo Insight. Fundación Universitaria de Ciencias de la Salud. Centro de Investigaciones de la Clínica del Country. Bogotá DC, Colombia.
*** Líder del grupo de Investigación en Patología. Fundación Universitaria de Ciencias de la Salud. Bogotá DC, Colombia.

**** Estudiante de Medicina. Fundación Universitaria de Ciencias de la Salud. Bogotá DC, Colombia. 
normal-appearing intermediate cells, three LSIL and three ASCUS). The fractal dimension of three mathematical objects: nuclei, cytoplasm and whole cells was calculated by means of a program based on the box-counting method; simultaneously determining the number of pixels occupied by the area of each of them and the spaces occupied by the outer margin of these objects in each of the five grids, to compare the obtained values. Results: when two and four pixel grids were superimposed on the cell, values of the occupied spaces of the nuclei allow mathematical differences to be established between groups of cells, presenting values in grid two: normal superficial (53-56), normal intermediate (75), LSIL (120-159) and ASCUS (104-121). Conclusions: a mathematical diagnostic methodology was developed differentiating pre-neoplastic states based on simultaneous fractal and Euclidian measures of the peripheral margin of the cell nuclei.

Key words: diagnostic, fractal, Euclidian, cytology, cervix.

\section{Introducción}

Las nomenclaturas para informes citológicos ginecológicos se basan en gran medida en observaciones cualitativas de la morfología celular. Dentro de ellas, es reconocida la clasificación o sistema Bethesda ${ }^{1}$, que permite catalogar las anormalidades celulares preneoplásicas mediante un sistema binario, denominando dichas anormalidades como lesiones intraepiteliales escamosas de alto o bajo grado. ${ }^{1}$ Pese a haber logrado un consenso, la clasificación Bethesda no ha sido adoptada en todos los países ${ }^{1}$, e incluye una zona gris denominada ASCUS: células escamosas atípicas de significado indeterminado. Son células que no tienen un adecuado diagnóstico y no se puede establecer cuáles evolucionarán a cáncer o bien volverán a presentar características de normalidad.

Las estructuras biológicas, como el cuerpo humano, se pueden caracterizar en forma adecuada mediante geometría fractal ${ }^{2}$, que se ha aplicado en diferentes áreas de la ciencia como morfología y fisiología animal y humana, economía, hidrología y geología, entre otros. $^{3-5}$ La GF surge como respuesta a la imposibilidad de realizar mediciones precisas de objetos naturales irregulares con la geometría euclidiana que mide longitudes, áreas y volúmenes de objetos regulares y homogéneos. ${ }^{6}$ Con ella es posible determinar el grado de irregularidad de un objeto, cuyo resultante es una medida numérica adimensional, denominada dimensión fractal. ${ }^{7}$ Existen diferentes formas de calcularla cuando es un objeto irregular. El fractal abstracto, cuya característica principal es la sibisemejanza o similaridad entre sus partes y la totalidad, se mide a partir de la dimensión de Hausdorff; el fractal salvaje, dado por la superposición entre sus partes, se mide mediante el método de box-counting ${ }^{8,9}$, por último, el fractal estadístico caracterizado por distribuciones hiperbólicas, que se mide mediante la ley de Zipf y Mandelbrot. ${ }^{10}$

En medicina el uso de dimensiones fractales ha permitido el desarrollo de importantes resultados tanto a nivel clínico como experimental, donde se ha efectuado la caracterización de objetos como la estructura neuronal y pulmonar, los intestinos, los vasos sanguíneos de diferentes estructuras, los árboles venosos y arteriales y la ramificación de las fibras del músculo cardíaco, al igual que otras redes como el árbol traqueobronquial y el sistema de conducción de His Purkinje. ${ }^{4-6,11,12}$

También ha sido aplicada con éxito en algunos estudios de cáncer, pues puede proporcionar medidas objetivas de diferenciación entre normalidad y enfermedad. ${ }^{13-19}$ Por ejemplo, Pohlman y col. ${ }^{15}$ y Lefebvre y Benali ${ }^{16}$, establecieron diferencias entre dimensiones fractales de imágenes mamográficas con lesiones neoplásicas y sin ellas, lo que permitiría la disminución de falsos positivos; Luzi y col. ${ }^{17}$ encontraron que las dimensiones fractales de muestras de cáncer invasivo de vejiga de alto grado presentaron valores mayores que las de bajo grado; Landini y Rippin ${ }^{18}$ realizaron un análisis multifractal del tejido conectivo de interfase de la mucosa oral en diferentes grados de lesión neoplásica, evidenciando espectros de valores para su discriminación. Gazit y col. ${ }^{3}$ encontraron diferencias matemáticas a nivel morfológico y fisiológico, entre la vasculatura tumoral y normal en tejido ocular de 
ratones en el desarrollo de neoplasias andrógeno dependientes.

La GF se ha aplicado en diversos fenómenos; por ejemplo en el área de la morfología y fisiología cardíaca se han diferenciado arterias coronarias de porcino normales y enfermas ${ }^{20}$; a partir de dichos resultados se realizó una generación teórica permitiendo establecer el número total de arterias normales y reestenosadas posibles. ${ }^{21}$ También se ha empleado en el desarrollo de metodologías diagnósticas en ecocardiografía pediátrica $^{22}$, la ramificación coronaria izquierda en agiografías $^{23,24}$ y el venticulograma izquierdo. ${ }^{25}$

Además se han realizado estudios de muestras citológicas de cuello uterino, desarrollando una caracterización matemática del grado de irregularidad de las células del epitelio escamoso identificadas al microscopio como normales, ASCUS y LEIBG. ${ }^{26}$ Luego se desarrolló una evaluación diagnóstica fractal ${ }^{27}$ donde se encontraron diferencias matemáticas entre células normales y L-SIL, diferenciando ASCUS que pueden tener las relaciones matemáticas de normalidad o de L-SIL. Hace poco se desarrolló una nueva metodología de investigación para células del epitelio escamoso del cuello uterino aplicable a la clínica, confirmando el trabajo desarrollado antes y hallando los prototipos geométricos celulares que representan a todas las posibles células que se pueden presentar en la práctica clínica, proporcionando una evaluación objetiva y reproducible que diferencia normalidad de lesiones preneoplásicas y carcinoma, aclarando con ayuda matemática el diagnóstico de células ASCUS. ${ }^{28}$

Sin embargo, se ha visto que en muchos casos las dimensiones fractales evaluadas de forma aislada no permiten diferenciar normalidad de enfermedad, como en el caso de la estructura eritrocitaria. Así se desarrolló una metodología que permite la diferenciación de eritrocitos normales y patológicos, de utilidad para la determinación de la viabilidad de bolsas de transfusión, mediante el uso simultáneo de geometría fractal y euclidiana. ${ }^{29}$ Este trabajo demostró que es posible lograr medidas objetivas y reproducibles de aplicación clínica mediante la unión de ambas geometrías, en casos donde el uso de solo una de ellas no permita el establecimiento de diferencias.
El propósito del presente trabajo es desarrollar un diagnóstico matemático de células de cuello uterino normales y preneoplásicas, mediante la aplicación simultánea de geometría fractal y euclidiana, a partir de las dimensiones fractales y los espacios de ocupación de la superficie y el borde de los objetos que componen las células.

\section{Metodología}

Definiciones: fractal: objeto irregular o irregularidad del mismo $^{2}$; dimensión fractal: medida numérica que evalúa la irregularidad de un objeto; para esta investigación se utilizó la de box-counting, empleada para fractales salvajes y descrita en la ecuación ${ }^{6}$ :

$$
D=\frac{\log N\left(2^{-(K+1)}\right)-\log N\left(2^{-K}\right)}{\log 2^{K+1}-\log 2^{K}}=\log _{2} \frac{N\left(2^{-(K+1)}\right)}{N\left(2^{-K}\right)}
$$

donde $\mathrm{N}$ es el número de cuadros que contiene el contorno del objeto, $\mathrm{K}$ el grado de partición de la cuadrícula y D la dimensión fractal; superficie del objeto, el número de pixeles ocupados por cada uno de los objetos definidos en cada célula (núcleo, citoplasma y totalidad); y borde del objeto, el número de cuadros ocupado por cada uno de los contornos de los objetos definidos en cada célula (núcleo, citoplasma y totalidad), con cada rejilla utilizada en el espacio de box counting.

\section{Procedimiento}

Este es un estudio en el que se tomaron en el servicio de patología del Hospital San José de Bogotá DC, muestras de citología de cuello uterino de mujeres con edades entre 20 y 55 años. Se seleccionaron doce células provenientes de las muestras citológicas: tres superficiales normales, tres intermedias normales, tres ASCUS y tres LEIBG. Los diagnósticos fueron realizados por un citólogo experto de acuerdo con los parámetros de evaluación actual.

Para la observación y toma de fotografías se utilizó un microscopio Leika Dm-2500, con un aumento de 100x. Se tomaron microfotografías digitales de las células 
de los extendidos, cuyas imágenes fueron llevadas a través de una interfase al computador, para ser analizadas por un editor de imágenes.

Empleando un programa (software) desarrollado antes, se calcularon las dimensiones fractales de tres objetos matemáticos definidos, que son: núcleo, citoplasma sin núcleo y totalidad de cada célula; para ello el programa superpone a cada imagen cinco rejillas de diferente magnitud (2, 4, 8, 16 y 32 pixeles), y cuantifica el número de cuadros que ocupa el borde de cada uno de los objetos medidos. Luego los valores hallados de los cuadros de ocupación retornan a la función que evalúa la dimensión fractal mediante el método de box-counting, usando la fórmula establecida en las definiciones. Se anotaron los valores del número de cuadros que son tocados por el borde de los objetos definidos en la rejilla utilizada y el número de pixeles que ocupa la superficie de cada uno de ellos. Por último se realizaron comparaciones entre las medidas obtenidas para la normalidad celular, tanto superficial como intermedia, así como para células ASCUS y LEIBG, en busca de posibles diferencias matemáticas de aplicabilidad diagnóstica.

\section{Resultados}

El análisis de los resultados mostró que las rejillas de mayor utilidad para la diferenciación del estado celular eran las de 2 y 4 pixeles, pues el nivel de escala de las restantes no permite evidenciar con claridad las diferencias en todos los casos. Dado que el mayor nivel de detalle es logrado con la de 2 pixeles, los resultados se presentarán a continuación sólo con esa rejilla, señalando los valores con la de 4 pixeles en los casos significativos.

Los valores del borde del núcleo en las rejillas (2 y 4) permiten establecer diferencias objetivas entre los grupos de células normales superficiales, las normales intermedias y las que presentaron LEIBG. Con las de 8,16 y 32 pixeles comienzan a presentarse superposiciones entre los valores de los grupos. Por ejemplo, en el caso de la rejilla 2, las cifras del borde del núcleo se encontraron entre 53 y 56 para las células superficiales normales, mientras que para las normales intermedias estas fueron siempre de 75 y para las células con LEIBG se encontraron entre 120 y 159. En cambio los valores de las células ASCUS están entre 104 y 121 y aunque en dos de los tres casos evaluados estos no se superponen con ninguno de los otros grupos, oscilan entre la normalidad de células intermedias y de LEIBG; en el caso de la célula AS2 se encuentra dentro del rango que caracterizó a las células con LEIBG, pero muy cerca al límite inferior con 121. En el caso de la rejilla 4, no se presentan superposiciones entre los rangos de ninguno de los grupos medidos, incluyendo el grupo de células ASCUS.

Los valores del borde del citoplasma en la rejilla 2 presentan diferencias entre normalidad, LEIBG y ASCUS, pero no se pueden diferenciar las células normales intermedias de las superficiales. Hubo valores entre 472 y 522 en células normales superficiales, entre 448 y 459 en normales intermedias, entre 411 y 528 en LEIBG y entre 616 y 808 en células ASCUS (Tablas 1 a 4$)$.

Los valores del borde de la totalidad con la rejilla 2 se encontraron entre 511 y 585 para células normales superficiales, entre 523 y 580 en normales intermedias, entre 561 y 656 para LEIBG y entre 732 y 935 en las células ASCUS, medidas que no permiten diferenciar entre los grupos (Tablas 1 a 4).

Las dimensiones fractales de los objetos componentes definidos en células normales superficiales se encontraron entre 0,936273 y 1,25845 , para las normales intermedias entre 0,943128 y 1,04576 , para LEIBG entre 0,93701 y 1,00523, y para ASCUS entre 0,953318 y 1,14321 , sin que se pudieran establecer rangos diferenciadores al comparar las medidas de núcleo, citoplasma y totalidad, entre los diferentes grupos (Tablas 1 a 4).

Los valores de la superficie evaluada en pixeles para el núcleo de células normales superficiales se encontraron entre 659 y 758, para normales intermedios se encontraron entre 1.327 y 1.487 , para LEIBG entre 2.890 y 5.396 y para células ASCUS entre 2.035 y 3.109 (Tablas 1 a 4). Se evidencia que la superficie del núcleo de las células superficiales es diferenciable de intermedias normales y de lesión de bajo grado pero se encuentra una superposición entre las cifras de ASCUS y LEIBG. 


\begin{tabular}{|c|c|c|c|c|c|c|c|c|c|c|}
\hline & \multirow[b]{2}{*}{ REJILLAS } & \multicolumn{3}{|c|}{$\mathbf{N} \mathbf{I}$} & \multicolumn{3}{|c|}{$\mathbf{N} \mathbf{2}$} & \multicolumn{3}{|c|}{ N3 } \\
\hline & & $\mathbf{N}$ & $\mathbf{T}$ & C & $\mathbf{N}$ & $\mathbf{T}$ & C & $\mathbf{N}$ & $\mathbf{T}$ & C \\
\hline \multirow[t]{5}{*}{ Borde } & 2 & 56 & 540 & 472 & 55 & 511 & 447 & 53 & 585 & 522 \\
\hline & 4 & 32 & 292 & 256 & 29 & 240 & 227 & 30 & 311 & 279 \\
\hline & 8 & 16 & 148 & 127 & 13 & 142 & 122 & 16 & 161 & 145 \\
\hline & 16 & 8 & 78 & 66 & 8 & 71 & 61 & 8 & 82 & 74 \\
\hline & 32 & 2 & 36 & 30 & 4 & 36 & 31 & 4 & 40 & 36 \\
\hline \multicolumn{2}{|c|}{ Dimensión fractal } & $1,16 \mid 47$ & 0,97182 & 0,9907II & 0,94207 & 0,96185 & 0,967602 & 0,936273 & I,25845 & 0,963063 \\
\hline \multicolumn{2}{|c|}{ Superficie } & 758 & 40933 & 39710 & 732 & 41935 & 40726 & 659 & 60043 & 58915 \\
\hline
\end{tabular}

\begin{tabular}{|c|c|c|c|c|c|c|c|c|c|c|}
\hline & \multirow[b]{2}{*}{ REJILLAS } & \multicolumn{3}{|c|}{ II } & \multicolumn{3}{|c|}{12} & \multicolumn{3}{|c|}{13} \\
\hline & & $\mathbf{N}$ & $\mathbf{T}$ & c & $\mathbf{N}$ & $\mathbf{T}$ & C & $\mathbf{N}$ & $\mathbf{T}$ & C \\
\hline \multirow[t]{5}{*}{ Borde } & 2 & 75 & 523 & 448 & 75 & 539 & 459 & 75 & 580 & 492 \\
\hline & 4 & 40 & 279 & 236 & 38 & 295 & 251 & 41 & 309 & 261 \\
\hline & 8 & 20 & 144 & 122 & 21 & $|5|$ & 128 & 22 & 160 & 137 \\
\hline & 16 & 10 & 75 & 63 & 9 & 74 & 64 & 10 & 78 & 66 \\
\hline & 32 & 4 & 37 & 33 & 5 & 36 & 30 & 4 & 41 & 35 \\
\hline \multicolumn{2}{|c|}{ Dimensión fractal } & I,04576 & 0,953773 & 0,943128 & 0,989178 & 0,980335 & 0,984246 & I,04933 & 0,963077 & 0,960997 \\
\hline \multicolumn{2}{|c|}{ Superficie } & 1327 & 42659 & 41180 & 1352 & 46477 & 44963 & 1487 & 49034 & 46891 \\
\hline
\end{tabular}

Tabla 3. Células con LEIBG: medidas de espacios de ocupación del borde con cada una de las rejillas, dimensión Fractal y superficie en pixeles de cada uno de los objetos

\begin{tabular}{|c|c|c|c|c|c|c|c|c|c|c|}
\hline & \multirow[b]{2}{*}{ REJILLAS } & \multicolumn{3}{|c|}{ L1 } & \multicolumn{3}{|c|}{ L2 } & \multicolumn{3}{|c|}{ L3 } \\
\hline & & $\mathbf{N}$ & $T$ & C & $\mathbf{N}$ & $T$ & C & $\mathbf{N}$ & $T$ & C \\
\hline \multirow[t]{5}{*}{ Borde } & 2 & 120 & 653 & 528 & 159 & 656 & 494 & 137 & 561 & 411 \\
\hline & 4 & 67 & 355 & 285 & 84 & 343 & 253 & 77 & 303 & 222 \\
\hline & 8 & 33 & 187 & 150 & 42 & 176 & 130 & 40 & 151 & 112 \\
\hline & 16 & 18 & 91 & 74 & 20 & 89 & 65 & 20 & 77 & 59 \\
\hline & 32 & 9 & 50 & 40 & 10 & 43 & 31 & 10 & 37 & 30 \\
\hline \multicolumn{2}{|c|}{ Dimensión fractal } & 0,93701 & 0,937805 & 0,93903 & 1,00523 & 0,980891 & 0,994897 & 0,949707 & 0,982119 & 0,946398 \\
\hline \multicolumn{2}{|c|}{ Superficie } & 2890 & 52416 & 48553 & 5396 & 45487 & 38930 & 4596 & 28364 & 22602 \\
\hline
\end{tabular}




\begin{tabular}{|c|c|c|c|c|c|c|c|c|c|c|}
\hline & \multirow[b]{2}{*}{ REJILLAS } & \multicolumn{3}{|c|}{ ASI } & \multicolumn{3}{|c|}{ AS2 } & \multicolumn{3}{|c|}{ AS3 } \\
\hline & & $\mathbf{N}$ & $\mathbf{T}$ & C & $\mathbf{N}$ & $\mathbf{T}$ & C & $\mathbf{N}$ & $\mathbf{T}$ & C \\
\hline \multirow[t]{5}{*}{ Borde } & 2 & 116 & 834 & 702 & 121 & 935 & 808 & 104 & 732 & 616 \\
\hline & 4 & 61 & 399 & 328 & 60 & 440 & 369 & 53 & 405 & 344 \\
\hline & 8 & 33 & 178 & 140 & 32 & 190 & 155 & 27 & 208 & 175 \\
\hline & 16 & 16 & 83 & 65 & 18 & 89 & 72 & 11 & 108 & 91 \\
\hline & 32 & 8 & 38 & 30 & 8 & 40 & 32 & 7 & 52 & 44 \\
\hline \multicolumn{2}{|c|}{ Dimensión fractal } & 0,96467 & 1,11772 & 1,14321 & 0,957469 & I,I3994 & $\mathrm{I}, 1674$ & $\mathrm{I}, 00547$ & $0,95374 \mid$ & 0,953318 \\
\hline \multicolumn{2}{|c|}{ Superficie } & 2685 & 146810 & 142593 & 3109 & 146579 & 142497 & 2035 & 93705 & 93705 \\
\hline
\end{tabular}

La superficie del citoplasma se encontró entre 39.710 y 58.915 para células superficiales normales, entre 41.180 y 46.891 para intermedias normales, entre 22.602 y 48.553 para LEIBG y entre 90.364 y 142.593 para ASCUS, medidas que no permiten diferenciar normalidad de LEIBG (Tablas 1 a 4).

La superficie de la totalidad de la célula presentó valores entre 40.933 y 60.043 para células superficiales normales, entre 42.659 y 49.034 para intermedias normales, entre 28.364 y 52.416 para LEIBG y entre 93.705 y 146.810 para las células ASCUS (Tablas 1 a 4). Se evidencia que es imposible diferenciar los rangos de normalidad y anormalidad.

Las proporciones del borde en su totalidad respecto al núcleo en la rejilla 2 se encontraron entre 9,290 y 11,037 en células superficiales normales, entre 6,973 y 7,733 en intermedias normales, entre 4,094 y 5,441 en LEIBG, y entre 7,038 y 7,727 en células ASCUS, evidenciándose que de este modo es posible diferenciar todos los grupos, con excepción de las células ASCUS respecto a las intermedias normales, cuyos valores se encuentran superpuestos. Esto mismo ocurre al comparar las proporciones del citoplasma respecto al núcleo y de la totalidad respecto al citoplasma (Tabla 5).

\section{Discusión}

Este es el primer trabajo en el que se realiza un diagnóstico matemático que permite la diferenciación objetiva y reproducible de células del cuello uterino normales superficiales, normales intermedias, ASCUS y con lesión intraepitelial de bajo grado, mediante el uso simultáneo de geometría fractal y euclidiana, aplicadas al núcleo celular, superando las clasificaciones basadas en parámetros cualitativos como el sistema Bethesda. ${ }^{1}$

\begin{tabular}{|c|c|c|c|c|}
\hline & & $\mathbf{T} / \mathbf{N}$ & $\mathbf{C} / \mathbf{N}$ & T/C \\
\hline \multirow{3}{*}{$\begin{array}{l}\text { Superficiales } \\
\text { normales }\end{array}$} & $\mathrm{NI}$ & 9,643 & 8,429 & $\mathrm{I}, 144$ \\
\hline & N2 & 9,291 & 8,127 & 1,143 \\
\hline & N3 & 11,038 & 9,849 & 1,121 \\
\hline \multirow{3}{*}{$\begin{array}{l}\text { Intermedias } \\
\text { normales }\end{array}$} & II & 6,973 & 5,973 & 1,167 \\
\hline & 12 & 7,187 & 6,120 & $\mathrm{I}, 174$ \\
\hline & 13 & 7,733 & 6,560 & 1,179 \\
\hline \multirow{3}{*}{ LEIBG } & LI & 5,442 & 4,400 & 1,237 \\
\hline & L2 & 4,126 & 3,107 & I,328 \\
\hline & L3 & 4,095 & 3,000 & 1,365 \\
\hline \multirow{3}{*}{ ASCUS } & ASI & 7,190 & 6,052 & 1,188 \\
\hline & AS2 & 7,727 & 6,678 & 1,157 \\
\hline & AS3 & 7,038 & 5,923 & 1,188 \\
\hline
\end{tabular}

El número de espacios de ocupación del borde del núcleo en las rejillas de 2 y 4 pixeles presenta cifras que diferencian con claridad cada estado, evidenciando un aumento del tamaño del borde celular a medida que progresa la lesión. De este modo no sólo se llega a un diagnóstico que aclara la indeterminación de las células ASCUS, obteniendo valores matemáticos que diferencian no solo el estado de normalidad (super- 
ficial e intermedia) o de LEIBG, sino que establece en forma cuantitativa la gravedad de la lesión para cualquier célula, con el aumento del valor de la medida del borde del núcleo, haciendo innecesario el uso de clasificaciones que utilicen parámetros cualitativos.

Aunque los valores matemáticos que permiten establecer el diagnóstico son los del borde del núcleo, se encontraron diferenciaciones significativas y de posible importancia clínica, que es necesario investigar a futuro. Tal es el caso de las cifras del borde del citoplasma en la rejilla 2, que diferencian estados de normalidad, LEIBG y ASCUS, pero no se pueden separar las células normales intermedias de las superficiales. Del mismo modo, la medida de superficie del núcleo caracteriza células normales superficiales, normales intermedias y LEIBG, pero los valores de las ASCUS se superponen a los de LEIBG. Al estudiar las proporciones de los bordes de las partes con la totalidad, se encontró que permiten diferenciar cada estado estudiado, excepto las células ASCUS de intermedias normales. Las medidas restantes no permiten establecer diferencias significativas.

De la misma forma que el diagnóstico realizado antes con base en geometría fractal y el concepto de variabilidad y AMI celular ${ }^{27,28}$, esta metodología diagnóstica establece diferencias objetivas y reproducibles entre normalidad y LEIBG, permitiendo además identificar células normales superficiales, intermedias y ASCUS. Es importante establecer en futuras investigaciones las implicaciones de la aplicación simultánea de la metodología diagnóstica desarrollada por Rodríguez y col. ${ }^{27,28}$ con base en medidas de variabilidad fractal y AMI celular, y las medidas obtenidas en el presente trabajo.

En el último siglo el cáncer de cérvix ha sido una de las patologías oncológicas más estudiadas, pero ha sido imposible establecer en forma cuantitativa las características de lesiones premalignas y malignas, por lo que el diagnóstico citológico es dependiente de la experiencia del observador, dificultando la unificación de los sistemas de clasificación y la emisión de conclusiones exactas durante el estudio patológico ${ }^{1,30}$, en especial en el paso desde normalidad a lo que en el sistema Bethesda se denomina LEIBG.
Uno de los parámetros evaluados en todos los sistemas es el aumento del tamaño del núcleo, asociado con la aparición de lesiones premalignas y malignas y que por lo regular se establece en forma cualitativa por el experto. ${ }^{1,30,31}$ Esta metodología permite la cuantificación objetiva de los cambios del núcleo, eliminando la subjetividad intrínseca de las evaluaciones cualitativas utilizadas en la actualidad, superando de este modo al sistema Bethesda.

La célula AS2 tuvo un borde cuyo valor en la rejilla de 2 pixeles se superpone con los valores de lesión de bajo grado, siendo muy cercana al límite inferior. Este hecho evidencia la dificultad matemática de establecer este tipo de diferenciaciones en forma cualitativa, lo que ha llevado a los expertos a continuar con la clasificación ASCUS en el sistema Bethesda, con el fin de delimitar la "zona gris" de indeterminación entre normalidad y lesiones de bajo grado. Dado el impacto clínico a nivel mundial del diagnóstico de estas células, es importante realizar más medidas con la metodología desarrollada, con un mayor número de células ASCUS, para confirmar las diferencias fundamentales y de este modo ratificar su aplicabilidad clínica. Del mismo modo, los resultados obtenidos deben usarse en futuros trabajos con células cancerígenas para ampliar el campo diagnóstico a cualquier grado de lesión incluyendo carcinoma.

En general, la hipótesis genética respecto al cáncer enuncia que los tumores son monoclonales, implicando que una masa tumoral se produce por la expansión clonal de una única célula progenitora que ha sufrido el daño genético, lo que significa que es necesaria una alteración del núcleo para el desarrollo tumoral. Al basarse en una forma de pensamiento matemática y acausal, esta metodología establece diferencias diagnósticas independientes de la etiología tumoral. En este sentido, esta nueva forma de medición del núcleo celular puede ser de utilidad para el diagnóstico de células preneoplásicas en cualquier tipo de tejido, por lo cual es importante realizar nuevos estudios en esta área.

La medida del borde celular es inadecuada solo con medidas euclidianas, por tratarse de una estructura irregular. Para lograrla fue necesario estudiar el contexto 
del espacio fractal de box counting, determinando el número de espacios ocupados en las diferentes escalas evaluadas. De este modo se obtuvo una medida euclidiana en un espacio fractal, simbiosis que permitió establecer diferencias diagnósticas de aplicabilidad clínica, contradiciendo la posición de Mandelbrot ${ }^{7}$, quien afirmó siempre que la geometría fractal y la euclidiana están separadas y no pueden unirse, por referirse a objetos de naturaleza y características completamente diferentes. Cabe destacar que el nacimiento mismo de la geometría fractal está asociada con la comparación con medidas euclidianas, señalando que no están separadas. ${ }^{8,9}$

El determinismo basado en la causalidad fue el método de investigación válido para cualquier área durante la era de la mecánica newtoniana, el cual fue cuestionado durante el desarrollo de la mecánica estadística ${ }^{32}$, las teorías del $\operatorname{caos}^{33}$ y la cuántica. ${ }^{34}$ Desde estas, la causalidad dejó de ser fundamento para entender la naturaleza y sus fenómenos. Desde esta perspectiva caótico determinista acausal, Prigogine ${ }^{35}$ considera que solo tenemos ventanas temporales para la evaluación de los fenómenos. Siguiendo esta línea, la presente metodología se desarrolló desde un punto de vista donde las relaciones causa efecto no se consideran, es por esto que es independiente de elementos externos como la edad, factores de riesgo y de cualquier análisis poblacional, pues lo que se observa son ventanas temporales de las células, revelando un orden armónico subyacente a la irregularidad fractal y a la linealidad euclidiana en forma simultánea con la estructura celular, estableciendo características evaluables matemáticas que permiten determinar parámetros objetivos, diferenciando normalidad y cada uno de los grados de lesión intraepitelial.

Aplicando el razonamiento seguido por la física teórica, en este trabajo se parte de pocas células, escogidas por ser características de cada uno de los estados estudiados, con el fin de desarrollar una inducción que permita establecer diferencias para cualquier célula cervical del universo definido. Este mismo tipo de pensamiento permitió no solo el logro de la metodología para identificar células preneoplásicas y neoplásicas basadas en dimensiones fractales ${ }^{27,28}$, sino también ha sido base para diagnósticos y predicciones de aplicabilidad clínica en todas las áreas de la medicina. Tal es el caso de la monitoría cardíaca fetal ${ }^{10}$ y la dinámica cardíaca del adulto, donde se logró diferenciar normalidad, enfermedad crónica y aguda, así como la evolución entre estos estados con base en la probabilidad y proporciones de la entropía ${ }^{36,37}$ y se estableció una ley exponencial que predice todas las posibles dinámicas cardíacas. ${ }^{38}$ En el área de inmunología se han expuesto teorías, caracterizaciones y predicciones de péptidos de unión. ${ }^{39,40}$ En el campo de la epidemiología se han desarrollado predicciones anuales de las dinámicas de malaria y de dengue y se realizó una predicción de brotes de malaria para grupos de tres semanas en 820 municipios de Colombia. ${ }^{41}$ En el área de la infectología se desarrolló una metodología predictiva de linfocitos T CD4, mediante la teoría de la probabilidad. ${ }^{42}$ Estos trabajos resaltan la importancia y aplicabilidad práctica de las medidas físicomatemáticas en la medicina, como el que se plantea en este trabajo.

\section{Limitaciones}

Existen varios factores inherentes a la técnica de la citología de cuello uterino que se deben tener en cuenta en la aplicación de la presente metodología, como los procesos de desecación y glicogenización que pueden modificar la relación núcleo/citoplasma simulando una célula ASCUS, la presencia de histiocitos y la técnica colorimétrica empleada.

Del mismo modo se deben tener presente los aspectos subyacentes a los cambios morfológicos celulares dependientes del ciclo hormonal natural y durante el climaterio, dado que pueden generar similitudes con células intermedias.

\section{Conclusiones}

Se desarrolló una metodología diagnóstica de las células de cuello uterino que diferencia las normales superficiales, normales intermedias, con LEIBG y ASCUS, mediante la aplicación simultánea de geometría fractal y euclidiana al borde del núcleo celular. Las medidas cuantifican la progresión de las alteraciones 
celulares hacia lesiones de mayor grado, con el aumento del valor del borde del núcleo, haciendo innecesario el uso de clasificaciones basadas en parámetros cualitativos como el sistema Bethesda. La metodología desarrollada define en forma cuantitativa el estado ASCUS, evidenciando por qué es tan difícil lograr un diagnóstico unívoco en observaciones cualitativas y proporcionando una medida objetiva y reproducible del estado celular aplicable a la clínica.

\section{Referencias}

1. Lacruz C. Nomenclatura de las lesiones cervicales (de Papanicolau a Bethesda 2001). Rev Esp Patol. 2003; 36(1):5-10.

2. Mandelbrot B. The Fractal Geometry of Nature. New York: W.H. Freeman 1972. p. 341-48.

3. Gazit Y, Baish JW, Safabaksh N. Fractal characteristics of tumor vascular architecture during tumor growth and regression. Microcirculation. 1997; 4:395-402.

4. Goldberger A, Rigney D, West B. Chaos and fractals in human physiology. Sci Am. 1990; 262:42-9.

5. Goldberger A. Non-linear dynamics for clinicians: chaos theory, fractals, and complexity at the bedside. Lancet. 1996; 347:1312-14

6. Mandelbrot B. ¿Cuánto mide la costa de Gran Bretaña?. En: Mandelbrot B. Los Objetos Fractales. Barcelona: Tusquets; 2000. p. 27, 50.

7. Mandelbrot B. Introducción. En: Mandelbrot B. Los Objetos Fractales. Barcelona: Tusquets Eds; 2000. p.13-26.

8. Peitgen O, Jürgens H, Dietmar S. Limits and self similarity. In: Chaos and fractals: new frontiers of science. New York: Springer-Verlag; 1992. p. 135-82.

9. Peitgen O, Jürgens H, Dietmar S. Length area and dimension. Measuring complexity and scalling properties. In: Chaos and fractals: new frontiers of science. New York: Springer-Verlag; 1992. p. 183-228.

10. Rodríguez J. Dynamical systems theory and ZIPF - Mandelbrot Law applied to the development of a fetal monitoring diagnostic methodology. XVIII Figo World Congress of Gynecology and Obstetric. Kuala Lumpur, Malaysia. Nov 2006.

11. West JW. Fractal physiology and chaos in medicine. Singapore: World Scientific; 1990.

12. Goldberger A, Amaral L, Hausdorff J, Ivanov P, Peng C, Stanley H. Fractal dynamics in physiology: alterations with disease and aging. Proc Natl Acad Sci U S A. 2002 Feb 19; 99 Suppl 1:2466-72.

13. Dey P, Rajesh L. Fractal dimension in endometrial carcinoma. Anal Quant Cytol Histol. 2004 Apr 26; 26(2):113-6.

14. Baish JW, Jain RK. Fractals and Cancer. Cancer Res. 2000 Jul 15; 60(14):3683-8.

15. Pohlman S, Powell KA, Obuchowski NA, Chilcote WA, Grundfest-Broniatowski S. Quantitative classification of breast tumors in digitized mammograms. Med Phys. 1996 Aug; 23(8):1337-45.

16. Lefebvre F, Benali H, Gilles R, Kahn E, Di Paola R. A fractal approach to the segmentation of microcalcifications in digital mammograms. Med Phys. 1995 Apr; 22(4):381-90.

17. Luzi P, Bianciardi G, Miracco C, Desanti MM, Del Vecchio MT, Alia L, Tosi P. Fractal analysis in human pathology. Ann NY Acad Sci. 1999 Jun; 879:255-57.

18. Landini G, Rippin JW. Fractal dimensions of epithelial-connective tissue interfaces in premalignant and malignant ephitelial lesions of the floor of mouth. Ana Quant Cytol Histol. 1993 Apr; 15(2):144-9.

19. Stehlík M, Mrkvička T, Filus J, Filus L. Recent developments on testing in cancer risk: a fractal and stochastic geometry. Journal of Reliability and Statistical Studies. 2012; 5 (Issue Special): 83-95.

20. Rodríguez J, Mariño M, Avilan N, Echeverri D. Medidas fractales de arterias coronarias, un modelo experimental en reestenosis armonía matemática intrínseca de la estructura arterial. Rev Col Cardiología. 2002; 10: 65-72.
21. Rodríguez J, Prieto S, Correa C, Bernal P, Puerta G, Vitery S, et al. Theoretical generalization of normal and sick coronary arteries with fractal dimensions and the arterial intrinsic mathematical harmony. BMC Medical Physics. 2010, 10:1.

22. Rodríguez J, Prieto S, Ortiz L, Ronderos M, Correa C. Diagnóstico Matemático de Ecocardiografías Pediátricas con Medidas de Dimensión Fractal evaluadas con Armonía Matemática Intrínseca. Rev colomb Cardiol. 2010; 17: 79-86.

23. Rodríguez J, Álvarez L, Mariño M, Avilán N, Prieto S, Casadiego E, et al. Variabilidad de la dimensión fractal del árbol coronario izquierdo en pacientes con enfermedad arterial oclusiva severa. Dinámica fractal de la ramificación coronaria. Rev Col Cardiol. 2004;11(4):185-192.

24. Rodríguez J, Prieto S, Ortiz L, Correa C, Álvarez L, Bernal P, et al. Variabilidad de la dimensión fractal de la ramificación coronaria izquierda en ausencia y presencia de enfermedad arterial oclusiva moderada y severa. Rev Colomb Cardiol. 2007; 14(3): 173-179.

25. Rodríguez J, Prieto S, Correa C, Bernal P, Álvarez L, Forero G, et al. Diagnóstico fractal del ventriculograma cardiaco izquierdo. Geometría fractal del ventriculograma durante la dinámica cardiaca. Rev Colomb Cardiol. 2012; 19(1):18-24.

26. Rodríguez J, Prieto S, Ortiz L, Wiesner C, Díaz M, Correa C. Descripción matemática con dimensiones fractales de células normales y con anormalidades citológicas de cuello uterino. Rev Cienc Salud. 2006; 4(2):58-63.

27. Rodríguez J. Nuevo método fractal de ayuda diagnóstica para células preneoplásicas del epitelio escamoso cervical. Rev UDCA Act Div Cient. 2011; 14(1):1522 .

28. Rodríguez J, Prieto S, Correa C, Posso H, Bernal P, Vitery S, et al. Generalización fractal de células preneoplásicas y cancerígenas del epitelio escamoso cervical de aplicación clínica. Rev Fac Med. 2010;18(2):173-181.

29. Correa C, Rodríguez J, Prieto S, Álvarez L, Ospino B, Munévar A, et al. Geometric diagnosis of erythrocyte morphophysiology. JMMS. 2012 Nov; 3(11): 715-720.

30. De Vita V Jr, Hellman S, Rosenberg S.Principios y practica de oncologia. $5^{\text {a }}$ ed. Bogotá: Medica Panamericana: ARAN ediciones; 1997. p. 3376.

31. Torroella M, Villa S. Bases Genéticas del Cáncer. México: Sección de Obras de Ciencia y Tecnología, 1998.

32. Fernandez - Rañada A. Movimiento caótico. En: orden y Caos. Barcelona: Scientific American. Prensa Científica, 1990. p. 66-77.

33. Crutchfield J, Farmer D, Packard N, Shaw R. Caos. En: orden y Caos. Barcelona: Scientific American; 1990. p. 78-90.

34. Feynman R. Leighton RB, Sands M. Comportamiento cuántico. En: Feynman R. Leighton RB, Sands M. Física. Wilmington: Addison-Wesley Iberoamericana; 1987.

35. Fernandez - Rañada A. Introducción. En: orden y Caos. Barcelona: Scientific American; 1990. p. 4-8.

36. Rodríguez J. Entropía proporcional de los sistemas dinámicos cardiacos: predicciones físicas y matemáticas de la dinámica cardiaca de aplicación clínica. Rev Colomb Cardiol. 2010; 17: 115-129.

37. Rodríguez J, Prieto S, Bernal P, Isaza D, Salazar G, Correa C, et al. Entropía proporcional aplicada a la evolución de la dinámica cardiaca. Predicciones de aplicación clínica. En: Comunidad del Pensamiento Complejo, editor. La emergencia de los enfoques de la complejidad en América Latina. En Prensa.

38. Rodríguez J. Mathematical law of chaotic cardiac dynamic: predictions of clinic application. J Med Sci. 2011; 2(8):1050-1059.

39. Rodríguez J, Bernal P, Prieto $\mathrm{S}$, Correa $\mathrm{C}$. Teoría de péptidos de alta unión de malaria al glóbulo rojo. Predicciones teóricas de nuevos péptidos de unión y mutaciones teóricas predictivas de aminoácidos críticos. Inmunología. 2010; 29(1):7-19.

40. Rodríguez J, Bernal P, Correa C, Prieto S, Benítez L, Vitery S, et al. Predicción de unión de péptidos de MSA-2 y AMA-1 de Plasmodium Falciparum al HLA clase II. Inmunología. 2009; 28(3):115-124.

41. Rodríguez J. Método para la predicción de la dinámica temporal de la malaria en los municipios de Colombia. Rev Panam Salud Pública. 2010;27(3):211-8.

42. Rodríguez J, Prieto S, Bernal P, Pérez C, Correa C, Álvarez L, et al. Predicción de la concentración de linfocitos T CD4 en sangre periférica con base en la teoría de la probabilidad. Aplicación clínica en poblaciones de leucocitos, linfocitos y CD4 de pacientes con VIH. Infectio. 2012; 16(1): 15-22. 